\title{
Non-toxic poly(ethylene terephthalate)/clay nanocomposites with enhanced barrier properties
}

\author{
Suren Hayrapetyan ${ }^{1}$, Antonios Kelarakis ${ }^{1}$, Luis Estevez, Qin Lin, Kausik Dana, Yi-Lin Chung, \\ Emmanuel P. Giannelis*
}

Department of Materials Science and Engineering, Cornell University, Ithaca, NY 14853, USA

\section{A R T I C L E I N F O}

\section{Article history:}

Received 18 October 2011

Received in revised form

4 December 2011

Accepted 10 December 2011

Available online 16 December 2011

\section{Keywords:}

Poly(ethylene terephthalate)

Barrier properties

Non-toxic

\begin{abstract}
A B S T R A C T
Motivated by the technological need for poly(ethylene terephthalate) materials with improved barrier properties together with the requirement for sustainability this study focuses on an eco-friendly sulfonated polyester as clay compatibilizer to facilitate polymer mixing during melt compounding. We demonstrate that the nanocomposites based on sulfonated polyester are a reliable alternative to their imidazolium counterparts, exhibiting enhanced properties (water vapor and UV transmission), without sacrificing the excellent transparency, clarity and mechanical strength of the matrix.
\end{abstract}

(c) 2011 Elsevier Ltd. All rights reserved.

\section{Introduction}

The structure and dynamics in clay nanocomposites strongly influence their macroscopic properties so that performance enhancements (thermomechanical, barrier, fire retardant efficiency) are directly related to the dispersion level of the filler and the strength of matrix-nanoparticle interactions [1-8]. Nanoclay hybrids can be prepared either by in situ polymerization, solution blending or direct melt intercalation, but only the latter method presents several advantages in terms of environmental impact (i.e. it eliminates the use of solvents), economical cost, preparation ease and compatibility with standard industrial processing [2]. For high melting point thermoplastics, the bottleneck of the method is the limited thermal stability of the organic components, e.g. the matrix itself and the clay modifier. Suffice to say that even a minimal degree of decomposition of the clay modifier during processing can have detrimental effects on the nanocomposites, not only by undermining intercalation, but also by inducing discoloration and loss of strength and clarity.

\footnotetext{
* Corresponding author.

E-mail addresses: epg2@cornell.edu, emmanuel@msc.cornell.edu (E.P. Giannelis).

1 Both authors contributed equally to the work.
}

To circumvent this problem the use of imidazolium and phosphonium, rather than ammonium, based clay modifiers has been proposed $[9,10]$. However, in view of the growing body of evidence about the (eco)toxicity of those compounds [11-13], serious concerns have been raised about their use with commodity polymers such as the poly(ethylene terephthalate) (PET). PET is commonly used as packaging material and is being in direct contact with food, beverages, pharmaceutical products and cosmetics.

Motivated by the technological need for improved barrier properties of PET materials (even a moderate improvement in barrier properties has a profound economic impact) and together with the requirement for sustainability, this study explores a nontoxic and eco-friendly sulfonated polyester (SPE) as clay compatibilizer. In particular, we focus on a commercial polyester of diethylene glycol with isophthalic and sulfoisophthalic acid that is an active ingredient in a variety of skin-care formulations [14]. For comparison, we also investigate the structure and properties of PET nanocomposites based on a thermally stable polymeric quaternized imidazolium clay modifier. Based on the Hazardous Materials Identification System scale the SPE and imidazolium considered here are rated 1 (slight hazard) and 3 (severe hazard), respectively [14]. We demonstrate that the nanocomposites based on the SPE modifier are reliable alternatives to their rather toxic imidazolium counterparts, exhibiting improved water vapor barrier properties and enhanced resistance to UV radiation, without any adverse effects on transparency, clarity and mechanical strength. 


\section{Experimental}

\subsection{Preparation of the nanocomposites}

\subsubsection{Materials}

PET (intrinsic viscosity $=0.82 \mathrm{dl} / \mathrm{g}$ ) was provided by Invista (grade 1103) and used as received. SPE (a polyester of diethylene glycol with isophthalic and sulfoisophthalic acid with $M_{n}=10^{5} \mathrm{~g} /$ mol, $T_{g}=35{ }^{\circ} \mathrm{C}$, intrinsic viscosity $=0.35 \mathrm{dl} / \mathrm{g}$, acid number $<2$, hydroxyl number $<10$ ) was provided as a $25 \mathrm{wt} \%$ aqueous dispersion by Eastman (grade AQ 38D). The ammonium-exchanged montmorillonite clays noted as MMT-Alk (I.30T from Nanocor Inc.) and MMT-OH (30B from Southern Clay Products) were functionalized with octadecyltrimethyl and bis(hydroxyethyl)methyl cations, respectively.

\subsubsection{Synthesis of the imidazolium based terpolymer (MSIm)}

The terpolymer was synthesized by free radical polymerization, following a protocol described elsewhere (except that methyl methacrylate was used instead of lauryl acrylate) [15]. A solution of styrene (St), methyl methacrylate (MMA), vinyl benzyl chloride (VBC) and azobisisobutyronitrile (AIBN) in tetrahydrofuran (THF) was refluxed under $\mathrm{N}_{2}$ for $12 \mathrm{~h}$ before being cooled down at room temperature. Addition of methanol resulted in copolymer precipitation that was subsequently washed with methanol and dried under vacuum. The copolymer was redissolved in THF, mixed with $\mathrm{N}$-methyl imidazole and refluxed under $\mathrm{N}_{2}$ for another $12 \mathrm{~h}$. A series of terpolymers were synthesized and evaluated (S.I. Scheme $1)$. The optimum composition in terms of thermal stability and miscibility with PET was found for $a=30, b=70, x=1$ (S.I. Fig. 1 ).

\subsubsection{Modification of montmorillonite clay}

A $10 \mathrm{wt} \%$ dispersion of SPE in water or $10 \mathrm{wt} \% \mathrm{MSIm}$ in THF was added dropwise and under vigorous stirring to a $1 \mathrm{wt} \%$ montmorillonite (MMT) clay suspension in water-isopropanol mixture (3:1 volume ratio). The modified clays (noted as MMT-SPE or MMTMSIm, respectively) were purified by repeated water washing/ centrifugation cycles and then freeze-dried.

\subsubsection{Preparation of the nanocomposites}

Prior to the preparation of the composites, all materials were dried to a vacuum oven overnight. The components were first thoroughly mixed in a Flack-Tek DAC-150 FV speed mixer, before being melt-extruded in a laboratory scale DSM twin screw microcompounder at $265{ }^{\circ} \mathrm{C}$ under flowing nitrogen (rotation speed $130 \mathrm{rpm}$, residence time $5 \mathrm{~min}$ ). The nanoclay content (including the organic modifier) was kept 5wt\% in all samples. Free-standing films and dumbbell shape specimens were prepared by compression molding at $290^{\circ} \mathrm{C}$. For comparison, unfilled polymer samples were prepared in an identical fashion. PET/MMT-SPE and PET/ MMT-MSIm refer to PET nanocomposites that contain $5 \mathrm{wt} \%$ MMT-SPE and MMT-MSIm clay, respectively.

\subsection{Methods}

Wide-angle X-ray diffraction (WAXS) spectra of the materials studied were recorded at room temperature using a Scintag Inc. $\theta-\theta$ goniometer (CuK $\alpha$ radiation, $\lambda=1.54 \AA$ ).

Thermogravimetric analysis (TGA) measurements were performed on a TGA Q 5000 with a heating rate of $10^{\circ} \mathrm{C} / \mathrm{min}$, scanning from room temperature up to $600{ }^{\circ} \mathrm{C}$ under flowing $\mathrm{N}_{2}$.

Differential Scanning Calorimetry (DSC) thermographs were collected by a TA Instrument Q1000 series calorimeter over the temperature range from 0 to $270{ }^{\circ} \mathrm{C}$ at a scan rate of $10^{\circ} \mathrm{C} / \mathrm{min}$. A heat/cool/heat protocol was followed, allowing $10 \mathrm{~min}$ at $270{ }^{\circ} \mathrm{C}$ and $0{ }^{\circ} \mathrm{C}$ at the end of the first heating and cooling scans, respectively.

Tensile tests were performed at room temperature with an Instron 5569 Mechanical Tester at constant strain rate of $5 \mathrm{~mm} / \mathrm{min}$. Dumbbell specimens with gauge length $29.5 \mathrm{~mm}$, width $4.0 \mathrm{~mm}$ and thickness $1.6 \mathrm{~mm}$ were used.

Transmission Electron Microscopy (TEM) images were obtained by A FEI T12 Spirit operated at $120 \mathrm{kV}$.

Water vapor transmission (WVT) tests were performed on uniform membranes (approximately $200 \mu \mathrm{m}$ thickness) sealed on the top of plastic cups that contained distilled water. Cups were left to equilibrate on a desiccator partially filled with saturated aqueous solution of $\mathrm{Mg}\left(\mathrm{NO}_{3}\right)_{2}$ to maintain humidity $53 \%$, while the temperature was kept within the range $20 \pm 2{ }^{\circ} \mathrm{C}$. The weight of the cups was recorded regularly. The WVT was calculated using the equation $\mathrm{WVT}=($ weight loss $\times$ thickness $) /($ time $\times$ film area $\times$ vapor pressure).

Ultraviolet (UV) transmission of thin films was measured at room temperature by a UV-3101 PC Shimadzu spectrometer.

\section{Results and discussion}

The TGA traces shown in Fig. 1 suggest that the clays modified with the sulfonated polyester (MMT-SPE) and the terpolymer modified imidazolium (MMT-MSIm) have organic content higher than $70 \mathrm{wt} \%$ compared to less than $25 \mathrm{wt} \%$ for the two representative ammonium-based clays (MMT-Alk and MMT-OH, see Experimental Section). This observation is a direct consequence of the polymeric nature of the modifiers in MMT-SPE and MMT-MSIm, as opposed to the low molecular weight surfactants present in MMTAlk and MMT-OH. The modification of clay with polycations or other polymers has been systematically explored as an effective route to well dispersed nanocomposites [16]. Particular emphasis has been given to polymers containing vinyl benzyl chloride repeating units that can be easily attached to an amine to form quaternary salts [17]. Interestingly, the use of polymeric and oligomeric modifiers from methyl methacrylate, styrene and vinyl benzyl chloride not only can facilitate efficient mixing with a variety of polymers, but also enhances the thermal stability of the clays [17]. To our knowledge, this is the first study on nanocomposites based on terpolymer modified imidazolium clays.

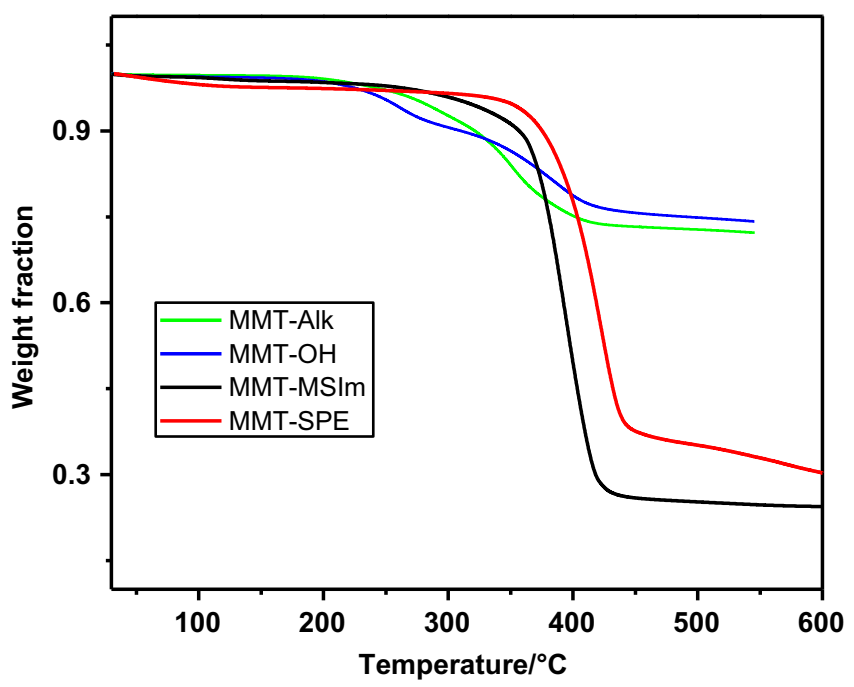

Fig. 1. TGA traces of MMT-SEI and MMT-MSIm compared to ammonium-based MMTAlk and MMT-OH clays. 


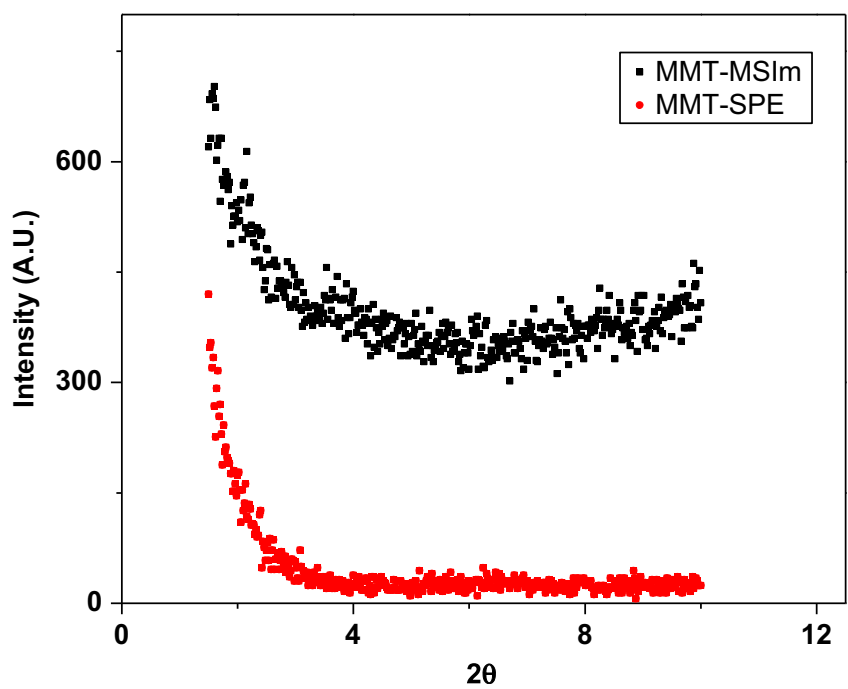

Fig. 2. XRD patterns of PET/MMT-SEI and PET/MMT-MSIm nanocomposites.

In addition, Fig. 1 shows that the decomposition onset temperature $\left(T_{\mathrm{dec}}\right)$ is close to $350{ }^{\circ} \mathrm{C}$ for MMT-SPE and $330{ }^{\circ} \mathrm{C}$ for MMTMSIm, but below $270{ }^{\circ} \mathrm{C}$ for MMT-Alk and MMT-OH. As a result, PET/MMT-SPE and PET/MMT-MSIm nanocompsites appear clear and thoroughly transparent, without any coloration (S.I. Fig. 1). In contrast, PET/MMT-Alk and PET/MMT-OH appear inhomogeneous, hazy and colored dark brown with dense black spots.

Given that the imidazole ring itself resists fission to temperatures up to $600{ }^{\circ} \mathrm{C}$ [18], it has been suggested that the decomposition of imidazolium salts commences through the thermal cleavage of the attached chains (via $\mathrm{S}_{\mathrm{N}} 1$ or $\mathrm{S}_{\mathrm{N}} 2$ mechanisms) within the temperature range $300-450{ }^{\circ} \mathrm{C}[19]$. On the other hand, the degradation of aromatic polyesters begins with the formation of cyclic oligomers and proceeds via chain scission due to a $\beta-\mathrm{C}-\mathrm{H}$ transfer reaction, generating vinyl esters and acid end groups [20]. Incorporation of ethylene glycol and isophthalate repeating units to aromatic polyesters (as in the case of the SPE modifier) increases the susceptibility to thermal degradation due to enhanced chain flexibility and the formation of more favorable bond angles, respectively, but even so the $T_{\mathrm{dec}}$ remains well-above $300{ }^{\circ} \mathrm{C}$ [21], consistent with the data shown in Fig. 1.

The featureless XRD patterns of PET/MMT-SPE and PET/MMTMSIm (Fig. 2) suggest the absence of a basal reflection of the clay tactoids, indicating sufficient matrix-filler mixing. TEM images of the PET/MMT-SPE and PET/MMT-Im nanocomposites at two different magnification scales are shown in Fig. 3a and b, respectively. At a large scale the TEM images of both nanocomposites indicate a rather patchy distribution of clay tactoids within the polymer matrix exhibiting morphological characteristics similar to those previously reported for a variety of PET/clay nanocomposites [22-27], including hybrids bearing imidazolium modified nanoclays [24].

Nevertheless, closer inspection of the TEM images reveals a certain level of clay intercalation and tactoids composed by a small number of silicate layers. Those morphological characteristics suggest the presence of rather favorable matrix-filler interactions due to the compatibilising efficiency of the clay modifiers. We note that the SPE modifier constitutes a polyester of isophalic acid and, thus, is structurally similar to the polyester of terephalic acid PET, while, at the same time, it contains negatively charged sulfonic groups that are known to exhibit strong affinity with the edges of the clay nanoparticles. It has been demonstrated that grafting of sulfonic groups along the PET backbone significantly enhances the interactions of the polymer with the positively charged edges of MMT platelets through strong electrostatic attraction [28]. Despite

a

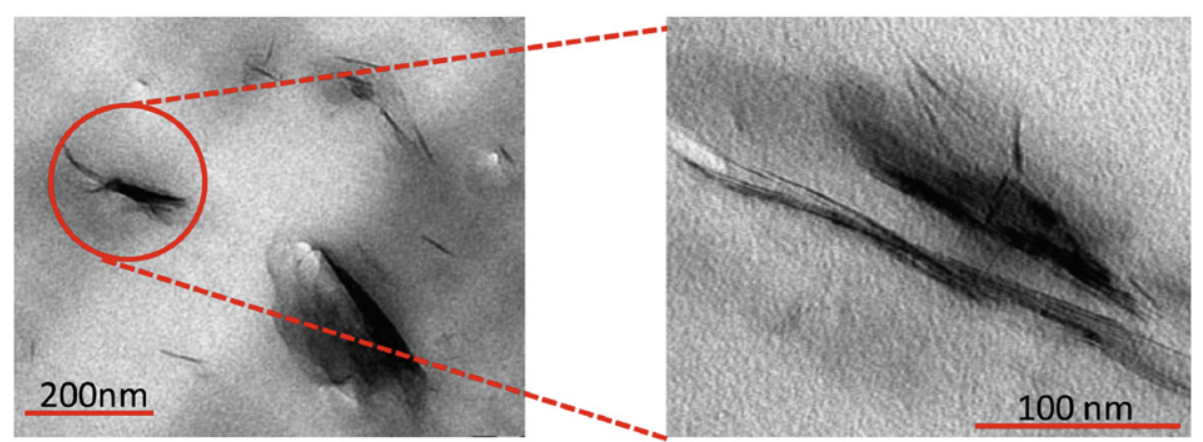

b

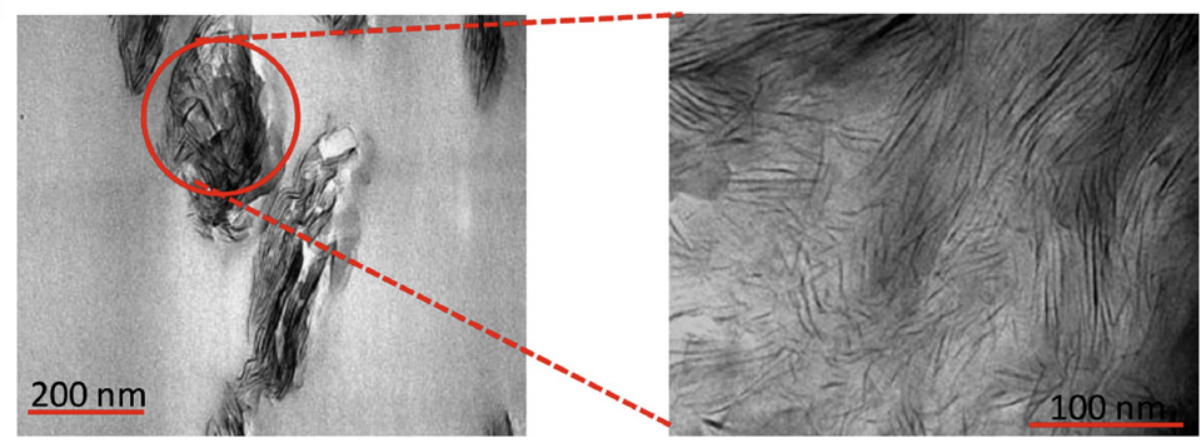

Fig. 3. TEM images of; a) PET/MMT-SEI and b) PET/MMT-MSIm nanocomposites. 
the inherent immiscibility of the PET/poly(methyl methacrylate) [29] and PET/ polystyrene [30] binary blends, the TEM images shown in Fig. 2b reveal the presence of extensive PET/MMT-MSIm interfaces. The TEM images reveal a remarkable high level of clay dispersion within the matrix, rarely achieved for melt processed PET nanocomposites.

Crystallization studies are crucial in understanding the macroscopic properties of nanocomposites (vide infra) given that inclusion of inorganic particles in polymeric matrices can induce diverse effects such as heterogeneous nucleation [31,32], suppression of crystal growth [33], and preferential development of a certain crystalline phase at the expense of other phases [34,35]. In agreement with previous studies on PET/clay systems [26,27,36] the DSC curves shown in Fig. 4 reveal the nucleating effect of MMT-SPE and MMT-MSIm clays as evident by the higher crystallization temperature $\left(T_{\mathrm{cr}}\right)$ observed for the nanocomposites compared to the neat matrix (cooling run in Fig. 4) and the position of the cold crystallization exotherms observed in the first heating run [36].

The multiple melting peaks oftentimes seen in neat PET have been attributed to melting/recrystallization/remelting sequences within a single heating run [37], to the development of a dual population of lamella thickness due to primary (thicker lamella) and secondary crystallization (thinner lamella) [38] or to a combination of those two contributions $[39,40]$. In Fig. 4 the double melting peaks observed during the 2nd heating for the two nanocomposites (but not for the neat PET) indicate that the clay nanoparticles induce the formation of smaller and imperfect crystallites by imposing steric constrains to their growth [23] or, alternatively, by facilitating the secondary crystallization. The enthalpy of fusion was $35 \mathrm{~J} / \mathrm{g}$ and $38 \mathrm{~J} / \mathrm{g}$ for the neat polymer and the nanocomposites, respectively, indicating that the clay nanoplatelets marginally increase the crystallinity of PET.

Addition of nanoclay leaves the Young's modulus (1.5 $\pm 0.1 \mathrm{GPa})$ and the elongation at break (340 $\pm 10 \%)$ unaffected (S.I. Fig. 2$)$. The minimal effect of clay addition suggests that the reinforcement expected due to the inclusion of rigid nanoparticles is counterbalanced by the formation of smaller and imperfect crystallites (vide supra).

Polymer-based clay nanocomposites often exhibit advanced barrier properties (for example gas permeation) in a manner that critically depends upon the volume fraction, the orientation

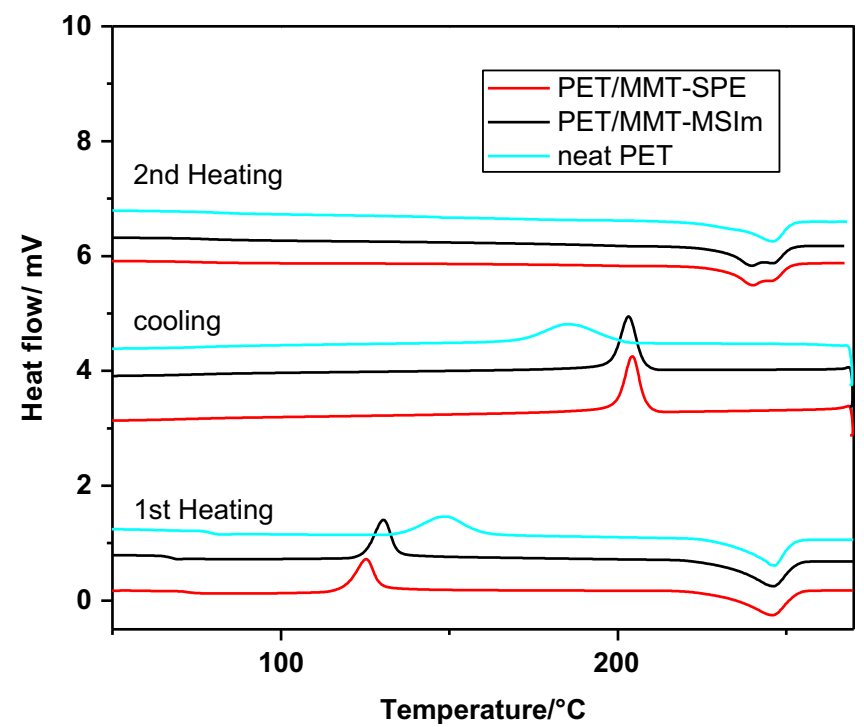

Fig. 4. DSC traces of neat PET, PET/MMT-SEI and PET/MMT-MSIm nanocomposites. relative to the diffusion direction and the aspect ratio of the nanoplatelets and the crystallinity of the matrix [41]. The clay nanoparticles function as impermeable physical barriers that increase the tortuosity and, by doing so, they slow down the diffusion of gases and liquids through a nanocomposite matrix. The effect can be compromised due to reduced crystallinity and increased free volume induced by the nanoparticles [42].

Ideally, packing materials (such as PET) should exhibit low water vapor transmission (WVT) in order to maintain certain moisture levels for susceptible products that are prone to dehydration or, oppositely, to damaging water absorption. For PET/clay nanocomposites it has been demonstrated that WVT cannot be easily controlled since it does not necessarily follow the same trends observed for the permeation of other gases. For example, addition of $5 \mathrm{wt} \%$ nanoclay to PET results in a remarkable improvement in $\mathrm{O}_{2}$ barrier properties by a factor of 15 , but the corresponding decrease in WVT was only 13\% [43]. Based on data plotted in Fig. 5 the WVT of the neat PET matrix considered here was $2.3 \times 10^{-12} \mathrm{~g} / \mathrm{m} \mathrm{s} \mathrm{Pa}$, in agreement with the value reported in literature [44]. Incorporation of either MMT-SEI or MMT-MSIm clay decreases the WVT by 22 and $30 \%$, respectively, compared to the neat PET membrane (Fig. 5).

Similar to WVT, the UV barrier characteristics of PET packing materials are equally important given that UV exposure can initiate photooxidative reactions, causing irreversible damages to the quality characteristics (e.g. nutrition or therapeutic value, color, odor) of susceptible products [45]. We found that the neat PET exhibit 75\% UV transmission at $370 \mathrm{~nm}$, and this value falls below $25 \%$ for the nanocomposites. In other words, addition of clay dramatically improves the UV shielding and also substantially lowers the WVT of the matrix, exhibiting performance enhancements highly desirable for packing applications.

In summary, we demonstrate that PET nanocomposites based on either polymeric imidazolium or sulfonated polyester modified clays lead to significant property improvements compared to the neat PET. In both cases intercalated hybrids that exhibit improved barrier properties, while retaining the excellent transparency and mechanical strength of the matrix are obtained. In addition, the sulfonated polyester modifier combines improved performance with environmentally friendly properties appropriate for food packaging and other health related applications.

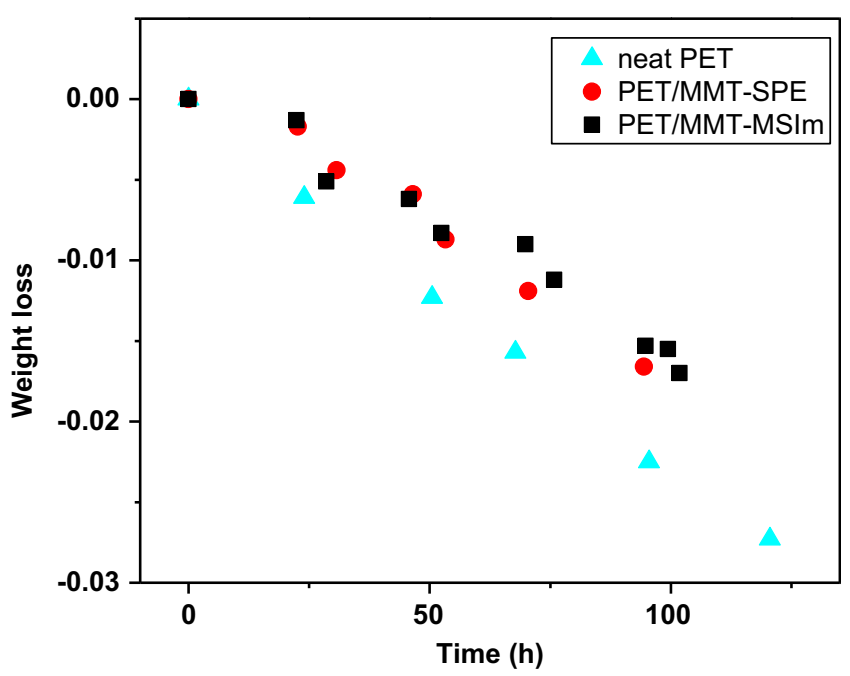

Fig. 5. Water vapor transmission of neat PET, PET/MMT-SEI and PET/MMT-MSIm nanocomposites films (room temperature, relative humidity $100 \%$ ). 


\section{Acknowledgment}

This material is based on work supported by Coca Cola. This publication is based on work supported in part by Award No. KUSC1-018-02, made by King Abdullah University of Science and Technology (KAUST).

\section{Appendix. Supplementary data}

Supplementary data associated with this article can be found, in the online version, at doi:10.1016/j.polymer.2011.12.017.

\section{References}

[1] Zeng OH, Yu AB, Lu GQ, Paul DR. J Nanosci Nanotechnol 2005;10:1574.

[2] Giannelis EP. Adv Mater 1996;8:29.

[3] Paul DR, Robenson LM. Polymer 2008;49:3187.

[4] Pavlidou S, Papaspyrides CD. Prog Polym Sci 2008;33:1119.

[5] Ray SS, Okamoto M. Prog Polym Sci 2003;28:1539.

[6] Yuan Q, Misra RDK. Mater Sci Technol 2006;22:742.

[7] Camargo PHC, Satyanarayana KG, Wypch F. Mater Res 2009;12:1.

[8] de Paiva LB, Morales AR, Diaz FRV. Appl Clay Sci 2008;42:8.

[9] Awad WH, Gilman JW, Nyden M, Harris RH, Sutto TE, Callahan J, et al. Thermochim Acta 2004;409:3.

[10] Calderon JU, Lennox B, Kamal MR. Appl Clay Sci 2008;40:90.

[11] Docherty KM, Kulpa CF. Green Chem 2005;7:185.

[12] Cho CW, Pham TPT, Jeon YC, Vijayaraghavan K, Choe WS, Yun YS. Chemosphere 2007;69:1003.

[13] Luo YR, Wang SH, Yun MX, Li XY, Wang IJ, Sun ZJ. Chemosphere 2009;77:313.

[14] Ash M, Ash I. Handbook of green chemicals. 2nd ed. New York: Synapse Information Resources; 2004. pp. 184.

[15] Zhang J, Jiang DD, Wilkie CA. Polym Degrad Stab 2006;91:641.

[16] Zhang J, Manias E, Wilkie CA. J Nanosci Nanotechnol 2008;8:1597.

[17] Leszczynska A, Njuguna J, Pielichowski, Banerjee JR. Thermochim Acta 2007; 453:75.
[18] Begg CG, Grimmett MR, Wethey PD. Aust J Chem 1973;26:2435.

[19] Ngo HG, LeCompte K, Hargens L, McEwen AB. Thermochim Acta 2000; 357-358:97.

[20] Montaudo G, Puglisi C, Samperi F. Polym Degrad Stab 1993;42:13.

[21] Holland BJ, Hay JN. Polymer 2002;43:1835.

[22] Ke YC, Long CF, Qi ZN. J Appl Polym Sci 1999;71:1139.

[23] Wan T, Chen L, Chua YC, Lu X. J Appl Polym Sci 2004;94:1381.

[24] Davis CH, Mathias LJ, Gilman JW, Schiraldi DA, Shields JR, Trulove P, et al J Polym Sci, Part B: Polym Phys 2002;40:2661.

[25] Costache MC, Heidecker MJ, Manias E, Wilkie CA. Polym Adv Technol 2006;17: 764.

[26] Calcagno CIW, Mariani CM, Teixeira SR, Mauler RS. Polymer 2007;48: 966.

[27] Guan G, Li C, Yuan X, Xiao Y, Liu X, Zhang D. J Polym Sci, Part B: Polym Phys 2008;46:2380.

[28] Barber GD, Calhoun BH, Moore RB. Polymer 2005;46:6706

[29] Bishara A, Shaban HI. J Appl Polym Sci 2006;101:3565.

[30] Ju MY, Chang FC. Polymer 2000;41:1719.

31] Jog JP. Mater Sci Technol 2006;22:797.

[32] Kelarakis A, Yoon K, Sics I, Somani RH, Chen X, Hsiao BS, et al. Macromol Sci Part B: Phys 2006;45:257.

[33] Kelarakis A, Giannelis EP. Polymer 2011;52:2221.

[34] Lincoln DM, Vaia RA, Wang ZG, Hsiao BS, Krishnamoorti R. Polymer 2001;42: 9975.

[35] Kelarakis A, Hayrapetyan S, Ansari S, Fang J, Estevez L, Giannelis EP. Polymer 2010;51:469.

[36] Durmus A, Ercan N, Soyubol G, Deligoz H, Kasgoz A. Polym Compos 2010;31: 1056.

[37] Jonas AM, Russell TP, Yoon DY. Colloid Polym Sci 1994;272:1344.

[38] Medellin-Rodriguez FJ, Phillips PJ, Lin JS. Macromolecules 1996;29:7491.

[39] Kong Y, Hay JN. Polymer 2003;44:623.

[40] Wang ZG, Hsiao BS, Sauer BB, Kampert WG. Polymer 1999;40:4615.

[41] Choudalakis G, Gotsis AD. Eur Polym J 2009;45:967-84.

[42] Cui L, Yeh JL, Wang K, Tsai FC, Fu Q. J Memb Sci 2009;327:226.

[43] Choi WJ, Kim HJ, Yoon KH, Kwon OH, Hwang CI. J Appl Polym Sci 2006;100: 4875.

[44] Auras RA, Singh SP, Singh JJ. Packag Technol Sci 2005;18:207.

[45] Tikekar RV, Anantheswaran RC, Elias RJ, Laborde LF. J Agric Food Chem 2011. 59:8244. 